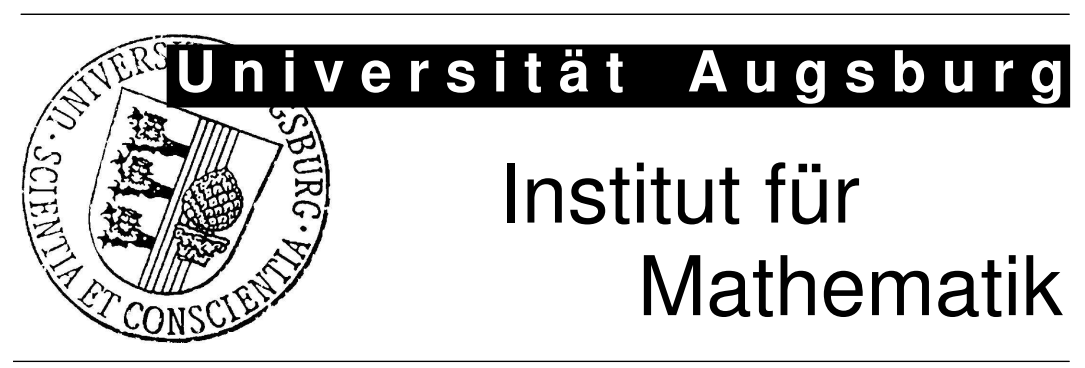

Christoph Kawan

Invariance Entropy of Control Sets

Preprint Nr. 01/2010 - 26. Januar 2010

Institut für Mathematik, Universitätsstraße, D-86135 Augsburg

http://www.math.uni-augsburg.de/ 


\section{Impressum:}

\section{Herausgeber:}

Institut für Mathematik

Universität Augsburg

86135 Augsburg

http://www.math.uni-augsburg.de/pages/de/forschung/preprints.shtml

\section{ViSdP:}

Christoph Kawan

Institut für Mathematik

Universität Augsburg

86135 Augsburg

Preprint: Sämtliche Rechte verbleiben den Autoren (C) 2010 


\title{
INVARIANCE ENTROPY OF CONTROL SETS
}

\author{
CHRISTOPH KAWAN*†
}

\begin{abstract}
Invariance entropy for continuous-time control systems measures how often openloop controls have to be updated in order to render a compact and controlled invariant subset of the state space invariant. A special type of a controlled invariant set for a control-affine system is the closure of a control set, i.e., a maximal set of approximate controllability. In this paper, we investigate the properties of the invariance entropy of such sets. Our main result gives an upper bound of this quantity in terms of the positive Lyapunov exponents of a periodic solution in the interior of the control set. Moreover, for one-dimensional systems with a single control vector field we provide an analytical formula for the invariance entropy of a control set in terms of the drift vector field, the control vector field and their derivatives. As an application, we study a controlled linear oscillator.
\end{abstract}

Key words. Nonlinear Control Systems, Invariance Entropy, Control Sets

AMS subject classifications. 93C15, 34C40, 94A17

1. Introduction. In [4, we introduced invariance entropy for continuous-time control systems on Euclidean space, to measure how often open-loop control functions have to be readjusted in order to stay in a compact and controlled invariant set $Q$ in the state space from a subset $K \subset Q$ of initial states. Precisely, we considered two quantities, the invariance entropy $h_{\text {inv }}(K, Q)$ and the strict invariance entropy $h_{\text {inv }}^{*}(K, Q)$, whereas in the definition of $h_{\text {inv }}(K, Q)$ we only required that trajectories stay in an arbitrarily small neighborhood of $Q$. In the $\mathrm{PhD}$ Thesis [6], we proved that the strict invariance entropy $h_{\mathrm{inv}}^{*}(Q)$ equals the infimal data rate necessary to render $Q$ invariant by a causal coding and control law. In [7, we extended the concept of invariance entropy to systems on arbitrary smooth manifolds and provided general upper and lower bounds. The present paper deals with control-affine systems on smooth manifolds and the invariance entropy of compact sets which are the topological closures of control sets, i.e., maximal sets of approximate controllability.

Let $M$ be a smooth manifold, endowed with a metric $d$ (not necessarily a Riemannian distance). On $M$ consider a control-affine system

$$
\dot{x}(t)=f_{0}(x(t))+\sum_{i=1}^{m} u_{i}(t) f_{i}(x(t)), \quad u \in \mathcal{U},
$$

with $L^{\infty}$-controls and a compact and convex control range $U \subset \mathbb{R}^{m}$. For simplicity, the vector fields $f_{0}, f_{1}, \ldots, f_{m}$ are assumed to be smooth and complete, guaranteeing that for any initial value $x \in M$ and control $u \in \mathcal{U}$ a unique solution $\varphi(t, x, u)$ exists for all $t \in \mathbb{R}$. By [5, Sec. 4.2 and 4.3] these assumptions imply compactness of $\mathcal{U}$ endowed with the weak*-topology of $L^{\infty}\left(\mathbb{R}, \mathbb{R}^{m}\right)=L^{1}\left(\mathbb{R}, \mathbb{R}^{m}\right)^{*}$, and continuity of the cocycle $\varphi: \mathbb{R} \times M \times \mathcal{U} \rightarrow M$. A control set $D \subset M$ of system (1.1) is a controlled invariant set such that for all $x, y \in D$ and $\varepsilon>0$ there are $u \in \mathcal{U}$ and $t>0$ with $d(\varphi(t, x, u), y)<\varepsilon$, and such that $D$ is maximal with these properties. In Section 4 , we will give the easy proof that controlled invariance of a set carries over to the closure of that set. Hence, if $D$ is a relatively compact control set, the (strict) invariance entropy $h_{\text {inv }}^{(*)}(K, \operatorname{cl} D)$ for each compact subset $K \subset \operatorname{cl} D$ is defined. The main lemma of this paper, Lemma

\footnotetext{
*christoph.kawan@math.uni-augsburg.de

${ }^{\dagger}$ Institut für Mathematik, Universität Augsburg, 86135 Augsburg/Germany
} 
4.2 states that $h_{\text {inv }}^{(*)}(K, \operatorname{cl} D)$ does actually not depend on the set $K$ as long as it is contained in $D$ and has nonvoid interior. The common value of $h_{\text {inv }}^{(*)}(K, \operatorname{cl} D)$ for $K$ with the described properties will simply be called the (strict) invariance entropy of the control set $D$. Using this lemma, we prove our main theorem, Theorem 4.4, which yields an upper bound for the strict invariance entropy of a control set in terms of the positive Lyapunov exponents of a periodic solution with controllable linearization in the interior of the control set. The proof of the theorem is a modification of the proof of a result by Nair, Evans, Mareels and Moran. In [10, they derived a formula for the local topological feedback entropy of a discrete-time control system in Euclidean space at a fixed point with controllable linearization in terms of the unstable eigenvalues of the fixed point Jacobian. Combining our main theorem with the lower estimate for invariance entropy, which we derived in [7, we are able to provide a formula for the invariance entropy of a control set for one-dimensional control-affine systems with a single control vector field. Moreover, we show that here $h_{\mathrm{inv}}(K, Q)$ and $h_{\mathrm{inv}}^{*}(K, Q)$ coincide. We apply this result to systems on the unit circle which are the projections of bilinear control systems in the plane. As a special case, we study a controlled mathematical pendulum, linearized at the unstable position. The first-order equations of such a pendulum can be regarded as a bilinear control system in the plane, which we project to the unit circle. Under suitable conditions, this projected system has two pairs of antipodal control sets, one pair of invariant and one of variant control sets. The double cone over the pair of variant control sets is exactly the region in the state space of the planar system, where stabilization at the unstable position is possible, and the number of control functions necessary to keep the planar system in that double cone for some positive time coincides with the number of control functions necessary to keep the projected system in the corresponding control sets. Hence, the invariance entropy of these control sets measures how often control functions have to be updated in order to stay in the region where stabilization is possible.

The present paper is organized as follows: In Section 2, we provide notation and recall facts on Riemannian manifolds, control-affine systems and invariance entropy. Section 3 introduces the linearization of a control system along a controlled trajectory. In Section 4, the central results are formulated and proved. Finally, Section 5 deals with the applications, including projected bilinear systems on the unit sphere andas a special case of that - a model for a damped mathematical pendulum linearized around the unstable equilibrium.

\section{Notation and Preliminaries.}

2.1. Notation. The term "smooth" always stands for $C^{\infty}$. By a smooth manifold we understand a connected, second-countable, topological Hausdorff manifold endowed with a smooth differentiable structure. A chart of a smooth $d$-dimensional manifold $M$ is a pair $(\phi, V)$ such that $V \subset M$ is an open set and $\phi$ is a diffeomorphism from $V$ onto an open subset of $\mathbb{R}^{d}$. By $T_{p} M$ we denote the tangent space of the manifold $M$ at $p \in M$, and by $T M$ the tangent bundle. For the space of smooth vector fields on $M$ we write $\mathcal{X}(M)$. A Riemannian manifold $(M, g)$ consists of a smooth manifold $M$ and a Riemannian metric $g$ on $M$, i.e., a family $\left(g_{p}\right)_{p \in M}$ of positive definite symmetric bilinear forms on $T_{p} M$, depending smoothly on $p$. By $\mathcal{L}(\gamma)$ we denote the length of a smooth curve $\gamma: I \rightarrow M$ on a Riemannian manifold.

If $(X, d)$ is a metric space, we write $B_{\varepsilon}(x)$ for the $\varepsilon$-ball centered at $x \in X$. We write int $A$ and $\operatorname{cl} A$ for the interior and the closure of a set $A \subset X$, respectively. The support of a continuous function $f: X \rightarrow \mathbb{R}$ is denoted by supp $f$. By $\sigma(A)$ 
we denote the spectrum of an endomorphism $A$. I stands for the identity matrix, and $(\cdot)^{T}$ for the transposed of a vector or a matrix. $\operatorname{Hom}(V, W)$ is the space of homomorphisms between the vector spaces $V$ and $W$. $\|\cdot\|$ denotes a vector or an operator norm. If $F(x, u)$ is a differentiable function of two arguments, we write $D_{1} F(x, u)$ or $D_{x} F(x, u)$ for the derivative with respect to the first, and $D_{2} F(x, u)$ or $D_{u} F(x, u)$ for the derivative with respect to the second argument, respectively. $\lfloor\cdot\rfloor$ denotes rounding down. Finally, we write $\|u\|_{[0, \tau]}$ for the $L^{\infty}$-norm of an essentially bounded measurable function $u:[0, \tau] \rightarrow \mathbb{R}^{m}$.

2.2. Riemannian Manifolds. Let $(M, g)$ be a Riemannian manifold with associated Levi-Civita connection $\nabla: \mathcal{X}(M) \times \mathcal{X}(M) \rightarrow \mathcal{X}(M),(g, f) \mapsto \nabla_{g} f$. Using the fact that $\left(\nabla_{g} f\right)(x)$ is independent of the values of $g$ except for $g(x)$, the covariant derivative of a vector field $f \in \mathcal{X}(M)$ at a point $x \in M$ is defined by

$$
\nabla f(x): T_{x} M \rightarrow T_{x} M, \quad v \mapsto\left(\nabla_{v} f\right)(x) .
$$

For every point $x \in M$ the Riemannian exponential function $\exp _{x}: T_{x} M \supset W \rightarrow M$ is defined on an open neighborhood $W$ of the origin $0 \in T_{x} M$ and has the property that $c(t):=\exp _{x}(t v)$ is the unique geodesic on $M$ such that $c(0)=x$ and $\dot{c}(0)=v$, where $v \in T_{x} M$. Moreover, $\exp _{x}$ is a local diffeomorphism around $0 \in T_{x} M$ and satisfies $D \exp _{x}(0)=\mathrm{id}_{T_{x} M}$.

2.3. Control-affine Systems. Let $M$ be a $d$-dimensional smooth manifold. A family of ordinary differential equations

$$
\dot{x}(t)=f_{0}(x(t))+\sum_{i=1}^{m} u_{i}(t) f_{i}(x(t)), \quad u \in \mathcal{U},
$$

on $M$ is called a control-affine system. Here $f_{0}, f_{1}, \ldots, f_{m} \in \mathcal{X}(M)$ are smooth and complete vector fields and the family of admissible control functions is given by

$$
\mathcal{U}=\left\{u: \mathbb{R} \rightarrow \mathbb{R}^{m}: u \text { measurable with } u(t) \in U \text { a.e. }\right\},
$$

where $U \subset \mathbb{R}^{m}$ is compact and convex. $f_{0}$ is also called the drift vector field and $f_{1}, \ldots, f_{m}$ the control vector fields of the system. We also denote the right-hand side by $F(x, u)$,

$$
F(x, u)=f_{0}(x)+\sum_{i=1}^{m} u_{i} f_{i}(x) .
$$

By smoothness of the vector fields $f_{0}, f_{1}, \ldots, f_{m}$, for every initial value $x \in M$ and every control function $u \in \mathcal{U}$ a unique solution $\varphi(\cdot, x, u)$ exists such that $\varphi(0, x, u)=x$. We also write $\varphi_{t, u}(x)$ or $\varphi_{t}(x, u)$ instead of $\varphi(t, x, u)$. Note that $\varphi(t, x, u)$ is also defined for an arbitrary $L^{\infty}$-function $u: \mathbb{R} \rightarrow \mathbb{R}^{m}$, and by completeness, all solutions are defined on $\mathbb{R}$. On $\mathcal{U}$ we introduce the shift flow $\left(\Theta_{t}\right)_{t \in \mathbb{R}}$ by

$$
\Theta: \mathbb{R} \times \mathcal{U} \rightarrow \mathcal{U}, \quad\left(\Theta_{t} u\right)(s):=u(t+s) .
$$

By [5, Lem. 4.3.2], both $\Theta$ and $\varphi$ are continuous and thus we obtain a continuous skew-product flow

$$
\Phi: \mathbb{R} \times(\mathcal{U} \times M) \rightarrow(\mathcal{U} \times M), \quad \Phi(t,(u, x))=\left(\Theta_{t} u, \varphi(t, x, u)\right),
$$


the so-called control flow of system 2.1. by

For a point $x \in M$ we define the set of points reachable from $x$ up to time $T>0$

$$
\mathcal{O}_{\leq T}^{+}(x):=\{y \in M \mid \exists u \in \mathcal{U}, t \in[0, T]: y=\varphi(t, x, u)\},
$$

and the set of points controllable to $x$ within time $T>0$,

$$
\mathcal{O}_{\leq T}^{-}(x):=\{y \in M \mid \exists u \in \mathcal{U}, t \in[0, T]: x=\varphi(t, y, u)\} .
$$

Furthermore, the positive and negative orbits of $x$ are defined by

$$
\mathcal{O}^{+}(x):=\bigcup_{T>0} \mathcal{O}_{\leq T}^{+}, \quad \mathcal{O}^{-}(x):=\bigcup_{T>0} \mathcal{O}_{\leq T}^{-}(x)
$$

System (2.1) is called locally accessible from $x \in M$ if both $\mathcal{O}_{\leq T}^{+}(x)$ and $\mathcal{O}_{\leq T}^{-}(x)$ have nonvoid interior for all $T>0$. The system is called locally accessible on a set $A \subset M$ if it is locally accessible from all $x \in A$.

A set $D \subset M$ is called a control set if it is maximal with the following properties:

(i) Controlled invariance: For all $x \in D$ there is $u \in \mathcal{U}$ with $\varphi\left(\mathbb{R}_{0}^{+}, x, u\right) \subset D$.

(ii) Approximate controllability: For all $x \in D$ it holds that $D \subset \operatorname{cl} \mathcal{O}^{+}(x)$.

Control sets with nonvoid interior have the so-called no-return property, i.e., if $x \in D$ and $\varphi(\tau, x, u) \in D$ for some $\tau>0$ and $u \in \mathcal{U}$, then $\varphi([0, \tau], x, u) \subset D$ (see [5, Prop. 1.3.8]).

By an equilibrium pair of system 2.1) we mean a pair $\left(x_{0}, u_{0}\right) \in M \times U$ such that $F\left(x_{0}, u_{0}\right)=0$, which is equivalent to $\varphi\left(t, x_{0}, u_{0}\right)=x_{0}$ for all $t \in \mathbb{R}$.

2.4. Invariance Entropy. Consider the control-affine system (2.1) and let $Q \subset$ $M$ be a compact and controlled invariant set. The latter means that for every $x \in Q$ there is $u \in \mathcal{U}$ with $\varphi\left(\mathbb{R}_{0}^{+}, x, u\right) \subset Q$. Moreover, let $K \subset Q$ be compact. A set $\mathcal{S} \subset \mathcal{U}$ is called $(T, \varepsilon, K, Q)$-spanning, where $T, \varepsilon>0$, if for all $x \in K$ there is $u \in \mathcal{S}$ with

$$
\varphi(t, x, u) \in N_{\varepsilon}(Q) \quad \text { for all } t \in[0, T]
$$

where

$$
N_{\varepsilon}(Q)=\{x \in M \mid \exists y \in Q: d(x, y)<\varepsilon\}
$$

is the $\varepsilon$-neighborhood of $Q$. Let $r_{\text {inv }}(T, \varepsilon, K, Q)$ be the minimal cardinality of such a set. Then the invariance entropy of $(K, Q)$ is defined by

$$
h_{\mathrm{inv}}(K, Q):=\lim _{\varepsilon \searrow 0} h_{\mathrm{inv}}(\varepsilon, K, Q), \quad h_{\mathrm{inv}}(\varepsilon, K, Q):=\limsup _{T \rightarrow \infty} \frac{1}{T} \ln r_{\mathrm{inv}}(T, \varepsilon, K, Q) .
$$

Existence of the limit easily follows from monotonicity of $r_{\text {inv }}(T, \varepsilon, K, Q)$ in $\varepsilon$, and finiteness of $r_{\text {inv }}(T, \varepsilon, K, Q)$ follows from compactness of $K$ and continuous dependence on initial conditions (see [4, Prop. 3.2(i)] or [6, Prop. 2.1.7]). Moreover, $h_{\text {inv }}(K, Q)$ does not depend on the metric $d$ imposed on $M$ (see [7, Prop. 6] or [6, Prop. 2.1.10(i)]).

A set $\mathcal{S}^{*} \subset \mathcal{U}$ is called $(T, K, Q)$-spanning if for all $x \in K$ there is $u \in \mathcal{S}^{*}$ with

$$
\varphi(t, x, u) \in Q \text { for all } t \in[0, T] .
$$


By $r_{\text {inv }}^{*}(T, K, Q)$ we denote the minimal cardinality of such a set, and we define the strict invariance entropy of $(K, Q)$ by

$$
h_{\text {inv }}^{*}(K, Q):=\limsup _{T \rightarrow \infty} \frac{1}{T} \ln r_{\text {inv }}^{*}(T, K, Q) .
$$

Here, existence of finite $T$-spanning sets is not guaranteed (see [6]). If there are no finite $(T, K, Q)$-spanning sets for some $T>0, h_{\text {inv }}^{*}(K, Q)$ is defined as $\infty$. Since every $(T, K, Q)$-spanning set is obviously $(T, \varepsilon, K, Q)$-spanning for all $\varepsilon>0$, the inequality

$$
h_{\mathrm{inv}}(K, Q) \leq h_{\mathrm{inv}}^{*}(K, Q)
$$

holds. In [4, we conjectured that here equality holds if $h_{\mathrm{inv}}^{*}(K, Q)<\infty$. In the present paper, we prove equality for control-affine systems with a single control vector field and one-dimensional state space, in the case that $Q$ is the closure of a control set (see Corollary 4.6).

The following proposition shows that for the computation of the invariance entropy it is sufficient to consider the system at times which are integer multiples of some fixed time step $\tau>0$. For a proof see [4, Prop. 3.4(ii)] or [6, Prop. 2.2.9].

Proposition 2.1. For all $\tau, \varepsilon>0$ it holds that

$$
h_{\text {inv }}(\varepsilon, K, Q)=\limsup _{\substack{n \rightarrow \infty \\ n \in \mathbb{N}}} \frac{1}{n \tau} \ln r_{\text {inv }}(n \tau, \varepsilon, K, Q),
$$

and

$$
h_{\mathrm{inv}}^{*}(K, Q)=\limsup _{\substack{n \rightarrow \infty \\ n \in \mathbb{N}}} \frac{1}{n \tau} \ln r_{\mathrm{inv}}^{*}(n \tau, K, Q)
$$

3. Linearization along Controlled Trajectories. In this section, we introduce the linearization of a control system along a controlled trajectory and show that the solutions of the linearization approximate the solutions of the nonlinear system in a neighborhood of the controlled trajectory. Moreover, we discuss controllability of the linearization along periodic trajectories.

Definition 3.1. Consider the control-affine system 2.1] and let $g$ be a Riemannian metric on $M$. Let $\varphi\left(\cdot, x_{0}, u_{0}\right): \mathbb{R} \rightarrow M$ be a solution corresponding to an initial value $x_{0} \in M$ and a control function $u_{0} \in \mathcal{U}$. Define

$$
\begin{aligned}
& A(t):=\nabla F_{u_{0}(t)}\left(\varphi_{t, u_{0}}\left(x_{0}\right)\right): T_{\varphi_{t, u_{0}}\left(x_{0}\right)} M \rightarrow T_{\varphi_{t, u_{0}}\left(x_{0}\right)} M, \\
& B(t):=D_{2} F\left(\varphi_{t, u_{0}}\left(x_{0}\right), u_{0}(t)\right): \mathbb{R}^{m} \rightarrow T_{\varphi_{t, u_{0}}\left(x_{0}\right)} M
\end{aligned}
$$

for all $t \in \mathbb{R}$. The pair $\left(\varphi\left(\cdot, x_{0}, u_{0}\right), u_{0}\right)$ is called a controlled trajectory and the family

$$
\frac{D z}{d t}(t)=A(t) z(t)+B(t) \mu(t), \quad \mu \in L^{\infty}\left(\mathbb{R}, \mathbb{R}^{m}\right),
$$

of differential equations, where $\frac{D}{d t}$ denotes the covariant derivative along the solution $\varphi\left(\cdot, x_{0}, u_{0}\right)$, is called the linearization of (2.1) along $\left(\varphi\left(\cdot, x_{0}, u_{0}\right), u_{0}\right)$. A solution of (3.1) with initial value $\lambda \in T_{x_{0}} M$ corresponding to a control function $\mu \in L^{\infty}\left(\mathbb{R}, \mathbb{R}^{m}\right)$ is a locally absolutely continuous vector field $z: I \rightarrow T M$ along $\varphi\left(\cdot, x_{0}, u_{0}\right)$, defined 
on an interval $I$ with $0 \in I$, satisfying equation (3.1) for almost all $t \in I$ such that $z(0)=\lambda$.

For the elementary but long and technical proof of the following proposition we refer to [6. Prop. 1.2.22]. Statements (i) and (ii) can be concluded from the corresponding "Euclidean version" (see, e.g., Theorem 1 in 9]) by writing everything in local coordinates. Statement (iii) immediately follows from (ii), and the identity in (iv) easily follows from periodicity and uniqueness of solutions.

Proposition 3.2. Consider the control-affine system (2.1). Let $\left(\varphi\left(\cdot, x_{0}, u_{0}\right), u_{0}\right)$ be a controlled trajectory with corresponding linearization (3.1). Then the following statements hold:

(i) For every $\tau>0$ the mapping

$$
\varphi_{\tau}: M \times L^{\infty}\left([0, \tau], \mathbb{R}^{m}\right) \rightarrow M, \quad(x, u) \mapsto \varphi(\tau, x, u),
$$

is continuously differentiable.

(ii) For every initial value $\lambda \in T_{x_{0}} M$ and every control function $\mu \in L^{\infty}\left(\mathbb{R}, \mathbb{R}^{m}\right)$ there exists a unique solution $\varphi^{l}(\cdot, \lambda, \mu): \mathbb{R} \rightarrow T M$ of 3.1 satisfying

$$
\varphi^{l}(t, \lambda, \mu)=D \varphi_{t}\left(x_{0}, u_{0}\right)(\lambda, \mu)
$$

for all $t \in \mathbb{R}$ and $(\lambda, \mu) \in T_{x_{0}} M \times L^{\infty}\left(\mathbb{R}, \mathbb{R}^{m}\right)$.

(iii) For every $\tau>0$ the mapping

$$
\varphi^{l}(\tau, \cdot, \cdot): T_{x_{0}} M \times L^{\infty}\left([0, \tau], \mathbb{R}^{m}\right) \rightarrow T_{\varphi\left(\tau, x_{0}, u_{0}\right)} M
$$

is linear and continuous.

(iv) Assume that the controlled trajectory $\left(\varphi\left(\cdot, x_{0}, u_{0}\right), u_{0}\right)$ is $T_{0}$-periodic for some $T_{0}>0$. Then for all $k \in \mathbb{Z}, t \in \mathbb{R}$ and $\lambda \in T_{x_{0}} M$ it holds that

$$
\varphi^{l}\left(t, \varphi^{l}\left(k T_{0}, \lambda, 0\right), 0\right)=\varphi^{l}\left(t+k T_{0}, \lambda, 0\right) .
$$

Statement (ii) of Proposition 3.2 shows that the linearization 3.1 is an object which actually does not depend on the Riemannian metric $g$ imposed on $M$, since the solutions are the same for every metric.

Proposition 3.3. Consider the control-affine system (2.1) and its linearization along the controlled trajectory $\left(\varphi\left(\cdot, x_{0}, u_{0}\right), u_{0}\right)$. Then for all $\tau, C>0$ there exist $\delta>0$ and a function $\zeta=\zeta_{\tau, C}:[0, \delta) \rightarrow \mathbb{R}_{0}^{+}$with

$$
\lim _{b \searrow 0} \zeta(b)=0
$$

such that

$$
\left\|\exp _{\varphi\left(\tau, x_{0}, u_{0}\right)}^{-1}(\varphi(\tau, x, u))-\varphi^{l}\left(\tau, \exp _{x_{0}}^{-1}(x), u-u_{0}\right)\right\| \leq \zeta(b) b
$$

for all $x \in M$ with $d\left(x, x_{0}\right)<b$ and $u \in L^{\infty}\left([0, \tau], \mathbb{R}^{m}\right)$ with $\left\|u-u_{0}\right\|_{[0, \tau]} \leq C b$, where $b \in[0, \delta)$ is small enough that $\exp _{x_{0}}^{-1}(x)$ and $\exp _{\varphi\left(\tau, x_{0}, u_{0}\right)}^{-1}(\varphi(\tau, x, u))$ are defined.

Proof. For given $\tau>0$ consider the mappings

$$
\alpha: M \times L^{\infty}\left([0, \tau], \mathbb{R}^{m}\right) \rightarrow M, \quad(x, u) \mapsto \varphi(\tau, x, u),
$$

and

$$
\begin{aligned}
& \widetilde{\alpha}: T_{x_{0}} M \times L^{\infty}\left([0, \tau], \mathbb{R}^{m}\right) \supset \widetilde{W} \\
&(y, u) \mapsto T_{\varphi\left(\tau, x_{0}, u_{0}\right)} M \\
& \exp _{\varphi\left(\tau, x_{0}, u_{0}\right)}^{-1}\left(\alpha\left(\exp _{x_{0}}(y), u\right)\right)
\end{aligned}
$$


where $\widetilde{W}$ is an open neighborhood of $\left(0, u_{0}\right) \in T_{x_{0}} M \times L^{\infty}\left([0, \tau], \mathbb{R}^{m}\right)$, chosen small enough such that $\widetilde{\alpha}$ is well-defined. Since $\alpha\left(\exp _{x_{0}}(0), u_{0}\right)=\varphi\left(\tau, x_{0}, u_{0}\right)$ and $\alpha$ is continuous, which follows from Proposition 3.2 (i), such $\widetilde{W}$ exists. By Proposition 3.2 (i) both $\alpha$ and $\widetilde{\alpha}$ are continuously differentiable. Differentiating $\widetilde{\alpha}$ at $\left(0, u_{0}\right)$ by the chain rule yields

$$
D \widetilde{\alpha}_{\left(0, u_{0}\right)}(\lambda, \mu)=D \exp _{\varphi\left(\tau, x_{0}, u_{0}\right)}^{-1}\left(\varphi\left(\tau, x_{0}, u_{0}\right)\right) D \alpha_{\left(x_{0}, u_{0}\right)} D\left(\exp _{x_{0}} \times \mathrm{id}\right)(\lambda, \mu) .
$$

Using that $D \alpha_{\left(x_{0}, u_{0}\right)}(\lambda, \mu)=\varphi^{l}(\tau, \lambda, \mu)$ (see Proposition 3.2(ii)) and that the derivative of the Riemannian exponential map at 0 is the identity, we obtain

$$
D \widetilde{\alpha}_{\left(0, u_{0}\right)}(\lambda, \mu)=\varphi^{l}(\tau, \lambda, \mu) .
$$

Thus,

$$
\begin{aligned}
\exp _{\varphi\left(\tau, x_{0}, u_{0}\right)}^{-1}\left(\varphi\left(\tau, \exp _{x_{0}}(y), u\right)\right) & =\widetilde{\alpha}(y, u) \\
& =\underbrace{\widetilde{\alpha}\left(0, u_{0}\right)}_{=0}+D \widetilde{\alpha}_{\left(0, u_{0}\right)}\left(y, u-u_{0}\right)+r(y, u) \\
& =\varphi^{l}\left(\tau, y, u-u_{0}\right)+r(y, u)
\end{aligned}
$$

for all $(y, u) \in \widetilde{W}$, where $r$ is a function that satisfies

$$
\lim _{(y, u) \rightarrow\left(0, u_{0}\right)} \frac{r(y, u)}{\|y\|+\|u\|_{[0, \tau]}}=0 .
$$

Hence, we obtain

$$
\left\|\exp _{\varphi\left(\tau, x_{0}, u_{0}\right)}^{-1}\left(\varphi\left(\tau, \exp _{x_{0}}(y), u\right)\right)-\varphi^{l}\left(\tau, y, u-u_{0}\right)\right\| \equiv\|r(y, u)\| .
$$

Since $\widetilde{W}$ is an open neighborhood of $\left(0, u_{0}\right)$, for given $C>0$ there exists $\delta>0$ such that $B_{\delta}(0) \times B_{C \delta}\left(u_{0}\right) \subset \widetilde{W}$. Define $\zeta_{\tau, C}:[0, \delta) \rightarrow \mathbb{R}_{0}^{+}$by

$$
\zeta_{C, \tau}(b):=\left\{\begin{array}{cc}
b^{-1} \sup _{\substack{\left.\|y\| \leq b,\left\|u-u_{0}\right\| \leq 0, \tau\right] \leq C b}}\|r(y, u)\| & \text { for } b \in(0, \delta), \\
0 & \text { for } b=0 .
\end{array}\right.
$$

Then from 3.5) we obtain (3.3). From (3.4) it follows that for every $\varepsilon>0$ there is $b>0$ such that $\|y\| \leq b$ and $\left\|u-u_{0}\right\|_{[0, \tau]} \leq C b$ implies $\frac{\|r(y, u)\|}{\|y\|+\|u\|_{[0, \tau]}} \leq \varepsilon$. Hence, from

$$
\frac{\|r(y, u)\|}{\|y\|+\|u\|_{[0, \tau]}}=\frac{\|r(y, u)\|}{b} \frac{b}{\|y\|+\|u\|_{[0, \tau]}} \leq \varepsilon
$$

it follows that

$$
\frac{\|r(y, u)\|}{b} \leq \varepsilon \frac{\|y\|+\|u\|_{[0, \tau]}}{b} \leq \varepsilon \frac{b(C+1)}{b}=\varepsilon(C+1) .
$$

For $b=b(\varepsilon)$ this implies

$$
\zeta_{C, \tau}(b)=\sup _{\substack{\|y\| \leq b \\\left\|u-u_{0}\right\|[0, \tau] \leq C b}} \frac{\|r(y, u)\|}{b} \leq \varepsilon(C+1),
$$


which finishes the proof.

Next, we introduce the notion of controllability for the linearization along a controlled periodic trajectory.

Definition 3.4. Let $\left(\varphi\left(\cdot, x_{0}, u_{0}\right), u_{0}\right)$ be a $T_{0}$-periodic controlled trajectory of system 2.1). Then the linearization along $\left(\varphi\left(\cdot, x_{0}, u_{0}\right), u_{0}\right)$ is called controllable (on $\left.\left[\mathbf{0}, \boldsymbol{T}_{\mathbf{0}}\right]\right)$ if for all $\lambda_{1}, \lambda_{2} \in T_{x_{0}} M$ there exists $\mu \in L^{\infty}\left(\left[0, T_{0}\right], \mathbb{R}^{m}\right)$ such that

$$
\varphi^{l}\left(T_{0}, \lambda_{1}, \mu\right)=\lambda_{2}
$$

or equivalently, if for each $\lambda \in T_{x_{0}} M$ there is $\mu \in L^{\infty}\left(\left[0, T_{0}\right], \mathbb{R}^{m}\right)$ with

$$
\varphi^{l}\left(T_{0}, \lambda, \mu\right)=0
$$

Proposition 3.5. Consider the control-affine system 2.1 and its linearization along a $T_{0}$-periodic controlled trajectory $\left(\varphi\left(\cdot, x_{0}, u_{0}\right), u_{0}\right)$. If the linearization is controllable, then there exists $C>0$ such that for all $\lambda \in T_{x_{0}} M$ there is $\mu \in$ $L^{\infty}\left(\left[0, T_{0}\right], \mathbb{R}^{m}\right)$ with

$$
\varphi^{l}\left(T_{0}, \lambda, \mu\right)=0 \text { and }\|\mu\|_{\left[0, T_{0}\right]} \leq C\|\lambda\| .
$$

Proof. By controllability, for every $\lambda \in T_{x_{0}} M$ there exists at least one $\mu \in$ $L^{\infty}\left(\left[0, T_{0}\right], \mathbb{R}^{m}\right)$ such that $\varphi^{l}\left(T_{0}, \lambda, \mu\right)=0$, or equivalently

$$
\varphi^{l}\left(T_{0}, 0, \mu\right)=\varphi^{l}\left(T_{0},-\lambda, 0\right)
$$

Consider the automorphism $Q: T_{x_{0}} M \rightarrow T_{x_{0}} M, Q \lambda:=\varphi^{l}\left(T_{0},-\lambda, 0\right)$, and the continuous linear operator

$$
L: L^{\infty}\left(\left[0, T_{0}\right], \mathbb{R}^{m}\right) \rightarrow T_{x_{0}} M, \quad \mu \mapsto \varphi^{l}\left(T_{0}, 0, \mu\right)
$$

Obviously, controllability is equivalent to $L$ being surjective. Hence, by the bounded inverse theorem (see [2, Theo. 16.5]), there exists a constant $\widetilde{C}>0$ such that for all $\lambda \in T_{x_{0}} M$ there is $\mu \in L^{\infty}\left(\left[0, T_{0}\right], \mathbb{R}^{m}\right)$ with $L \mu=Q \lambda$ and $\|\mu\|_{\left[0, T_{0}\right]} \leq \widetilde{C}\|Q \lambda\|$. Thus, with $C:=\widetilde{C}\|Q\|$ the assertion holds.

REMARK 3.1. For control systems on Euclidean space our notion of controllability for the linearization along a (periodic) controlled trajectory coincides with the usual one, as it is defined, e.g., in [9]. In particular, in the case of an equilibrium pair $\left(x_{0}, u_{0}\right)$, controllability is equivalent to controllability of the matrix pair $(A, B)$ given by $A=D_{x} F\left(x_{0}, u_{0}\right)$ and $B=D_{u} F\left(x_{0}, u_{0}\right)$.

4. The Main Results. In this section, we state and prove our main results about the invariance entropy of control sets. First, we prove the following easy lemma, which shows that controlled invariance of a set carries over to the closure of that set. This guarantees that $h_{\text {inv }}^{(*)}(K, \mathrm{cl} D)$ is a well-defined quantity if $D$ is a relatively compact control set and $K \subset D$ is compact.

LEMma 4.1. Consider the control-affine system 2.1 and let $A \subset M$ be a controlled invariant set. Then also $\mathrm{cl} A$ is controlled invariant.

Proof. Let $x \in \operatorname{cl} A$ be chosen arbitrarily. Then there exists a sequence $\left(x_{n}\right)_{n \in \mathbb{N}}$ in $A$ with $x_{n} \rightarrow x$. By controlled invariance, there are $u_{n} \in \mathcal{U}$ with $\varphi\left(\mathbb{R}_{0}^{+}, x_{n}, u_{n}\right) \subset A$ for all $n \geq 1$. By compactness of $\mathcal{U}$ (in the weak*-topology) we may assume that 
$u_{n} \rightarrow u \in \mathcal{U}$. By continuity of $\varphi$ we have $\varphi\left(t, x_{n}, u_{n}\right) \rightarrow \varphi(t, x, u)$ for each $t \geq 0$, which implies $\varphi\left(\mathbb{R}_{0}^{+}, x, u\right) \subset \operatorname{cl} A$. Hence, the assertion holds.

Next we prove our main lemma, which justifies to speak of the (strict) invariance entropy of a control set.

LEMMA 4.2. Let $D$ be a control set of the control-affine system (2.1) with compact closure $Q=\operatorname{cl} D$ and nonvoid interior. Further, let $K_{1}, K_{2} \subset D$ be compact sets with nonvoid interior. Then

$$
h_{\mathrm{inv}}\left(K_{1}, Q\right)=h_{\mathrm{inv}}\left(K_{2}, Q\right) \text { and } h_{\mathrm{inv}}^{*}\left(K_{1}, Q\right)=h_{\mathrm{inv}}^{*}\left(K_{2}, Q\right) .
$$

Proof. Consider the first equality. Obviously, it suffices to prove that the left-hand side is less than or equal to the right-hand side. By approximate controllability on $D$, we can assign to each $x \in K_{1}$ a control function $u_{x} \in \mathcal{U}$ and a time $t_{x} \geq 0$ such that $\varphi\left(t_{x}, x, u_{x}\right) \in \operatorname{int} K_{2}$. By the no-return property, we also have $\varphi\left(\left[0, t_{x}\right], x, u_{x}\right) \subset D$. By continuous dependence on initial conditions, one finds a neighborhood $V_{x}$ of $x$ with $\varphi\left(t_{x}, V_{x}, u_{x}\right) \subset \operatorname{int} K_{2}$. The family $\left\{V_{x}\right\}_{x \in K_{1}}$ is an open cover of $K_{1}$. By compactness, there exist $x_{1}, \ldots, x_{n} \in K_{1}$ with $K_{1} \subset \bigcup_{i=1}^{n} V_{x_{i}}$. Now, for arbitrary $T, \varepsilon>0$ let $\mathcal{S}=\left\{v_{1}, \ldots, v_{k}\right\}$ be a minimal $\left(T, \varepsilon, K_{2}, Q\right)$-spanning set. For every index pair $(i, j)$ with $1 \leq i \leq n$ and $1 \leq j \leq k$ such that there exists $x \in K_{1}$ with $y_{x}:=\varphi\left(t_{x_{i}}, x, u_{x_{i}}\right) \in$ int $K_{2}$ and $\varphi\left([0, T], y_{x}, v_{j}\right) \subset N_{\varepsilon}(Q)$ we define the control function $w_{i j}$ by

$$
w_{i j}(t):= \begin{cases}u_{x_{i}}(t) & \text { for } t \in\left[0, t_{x_{i}}\right] \\ v_{j}\left(t-t_{x_{i}}\right) & \text { for } t>t_{x_{i}}\end{cases}
$$

The number of these control functions is bounded from above by $n k=n r_{\mathrm{inv}}\left(T, \varepsilon, K_{2}, Q\right)$. Consider the set $\widehat{\mathcal{S}}$ consisting of the control functions $w_{i j}$. Let $\widehat{T}:=T+\min _{i=1, \ldots, n} t_{x_{i}}$. Then, by construction, $\widehat{\mathcal{S}}$ is a $\left(\widehat{T}, \varepsilon, K_{1}, Q\right)$-spanning set. Consequently,

$$
r_{\mathrm{inv}}\left(T, \varepsilon, K_{1}, Q\right) \leq r_{\mathrm{inv}}\left(\widehat{T}, \varepsilon, K_{1}, Q\right) \leq n r_{\mathrm{inv}}\left(T, \varepsilon, K_{2}, Q\right) .
$$

Hence, we obtain

$$
h_{\mathrm{inv}}\left(\varepsilon, K_{1}, Q\right) \leq \limsup _{T \rightarrow \infty}\left(\frac{\ln n}{T}+\frac{\ln r_{\mathrm{inv}}\left(T, \varepsilon, K_{2}, Q\right)}{T}\right)=h_{\mathrm{inv}}\left(\varepsilon, K_{2}, Q\right) .
$$

For $\varepsilon \searrow 0$, the desired inequality $h_{\text {inv }}\left(K_{1}, Q\right) \leq h_{\text {inv }}\left(K_{2}, Q\right)$ follows. The proof for the strict invariance entropy works analogously. $\square$

For the proof of our main theorem we also need the following well-known property of the matrix exponential, which can easily be concluded from [8, Theo. 5.1], for instance.

Lemma 4.3. Let $A \in \mathbb{R}^{d \times d}$ and denote by $\alpha(A)$ the maximum of the real parts of all eigenvalues of $A$. Then

$$
\forall \delta>0: \exists c>0: \forall t \geq 0:\left\|e^{A t}\right\| \leq c e^{(\alpha(A)+\delta) t},
$$

where $\|\cdot\|$ denotes any operator on $\mathbb{R}^{d \times d}$.

Now we are able to prove our main theorem, whose proof is a modification of the second part of the proof of Theorem 3 in [10, which states that the local topological feedback entropy of a discrete-time system $x_{k+1}=F\left(x_{k}, u_{k}\right)$ at an equilibrium pair $\left(x_{*}, u_{*}\right)$ is given by $\sum_{\eta \in \sigma(A)} \max \left\{0, \log _{2}|\eta|\right\}$, where $A=D_{x} F\left(x_{*}, u_{*}\right)$ and $B=D_{u} F\left(x_{*}, u_{*}\right)$.

THEOREM 4.4. Let $D$ be a control set of the control-affine system (2.1) with nonvoid interior and compact closure $Q:=\mathrm{cl} D . \operatorname{Let}\left(\varphi\left(\cdot, x_{0}, u_{0}\right), u_{0}\right)$ be a $T_{0}$-periodic 
controlled trajectory with $\varphi\left(\left[0, T_{0}\right], x_{0}, u_{0}\right) \subset$ int $D$. Moreover, assume that $u_{0}(t)$ is contained in a compact subset of $\operatorname{int} U$ for almost all $t \in\left[0, T_{0}\right]$, and that the linearization of (2.1) along $\left(\varphi\left(\cdot, x_{0}, u_{0}\right), u_{0}\right)$ is controllable. Let $\rho_{1}, \ldots, \rho_{r}$ be the different Lyapunov exponents of the solution $\varphi\left(\cdot, x_{0}, u_{0}\right)$ with corresponding multiplicities $d_{1}, \ldots, d_{r}$. Then for every compact set $K \subset D$ it holds that

$$
h_{\mathrm{inv}}^{*}(K, Q) \leq \sum_{j=1}^{r} d_{j} \max \left\{0, \rho_{j}\right\} .
$$

Proof. We prove the theorem in three steps.

Step 1: Consider the automorphism

$$
A:=D \varphi_{2 T_{0}}\left(x_{0}, u_{0}\right)(\cdot, 0) \stackrel{[3.2}{=} \varphi^{l}\left(2 T_{0}, \cdot, 0\right): T_{x_{0}} M \rightarrow T_{x_{0}} M .
$$

By Proposition 3.2 (iv) it holds that $A=\varphi^{l}\left(T_{0}, \cdot, 0\right)^{2}$ and hence from the fundamental lemma of Floquet theory (see, e.g., [3, Theo. 2.4.7]) it follows that there exists a linear endomorphism $R: T_{x_{0}} M \rightarrow T_{x_{0}} M$ with

$$
A=e^{2 T_{0} R} .
$$

By Proposition 3.2(iv) it follows that

$$
\varphi^{l}\left(2 T_{0} k, \lambda, 0\right)=A^{k} \lambda=e^{2 T_{0} k R} \lambda \text { for all } \lambda \in T_{x_{0}} M, k \in \mathbb{N} .
$$

The real parts of the eigenvalues of $R$ are exactly the Lyapunov exponents of the solution $\varphi\left(\cdot, x_{0}, u_{0}\right)$. To show this, we write $t>0$ as $t=2 T_{0} k+s$ with $k \in \mathbb{N}_{0}$ and $s \in\left[0,2 T_{0}\right)$. Then for all $\lambda \in T_{x_{0}} M$ we obtain

$$
\varphi^{l}(t, \lambda, 0)=\varphi^{l}\left(s, \varphi^{l}\left(k\left(2 T_{0}\right), \lambda, 0\right), 0\right) \stackrel{(4.4)}{=} \varphi^{l}(s, \cdot, 0) e^{2 T_{0} k R} \lambda .
$$

Hence,

$$
l_{1}\left\|e^{2 k T_{0} R} \lambda\right\| \leq\left\|\varphi^{l}(t, \lambda, 0)\right\| \leq l_{2}\left\|e^{2 k T_{0} R} \lambda\right\|
$$

with the positive constants

$$
l_{1}:=\min _{s \in\left[0,2 T_{0}\right]}\left\|\varphi^{l}(s, \cdot, 0)^{-1}\right\|^{-1}, \quad l_{2}:=\max _{s \in\left[0,2 T_{0}\right]}\left\|\varphi^{l}(s, \cdot, 0)\right\| .
$$

By Proposition 3.2(ii) we have $D \varphi_{t, u_{0}}\left(x_{0}\right) \lambda=\varphi^{l}(t, \lambda, 0)$ and hence the exponential growth rate of $\left\|D \varphi_{t, u_{0}}\left(x_{0}\right) \lambda\right\|$ for $t \rightarrow \infty$ equals the growth rate of $\left\|e^{2 T_{0}\left\lfloor\frac{t}{2 T_{0}}\right\rfloor R} \lambda\right\|$ for all $\lambda \in T_{x_{0}} M$, which proves the claim.

Choose a basis $B_{x_{0}}$ of $T_{x_{0}} M$ adapted to the real Jordan structure of $R$. Let $L_{1}(R), \ldots, L_{r}(R)$ be the different Lyapunov spaces of $R$. Then we have the decomposition

$$
T_{x_{0}} M=L_{1}(R) \oplus \cdots \oplus L_{r}(R) .
$$

Let $d_{j}=\operatorname{dim} L_{j}(R)$ and denote by $\lambda^{(j)} \in L_{j}(R)$ the $j^{\text {th }}$ component of a vector $\lambda \in T_{x_{0}} M$ with respect to this decomposition. Moreover, denote by $\rho_{j}$ the common real part of the eigenvalues corresponding to $L_{j}(R)$. For the restriction of $R$ to $L_{j}(R)$ 
we write $R_{j}$. Now, let $g$ be a Riemannian metric on $M$ such that the basis $B_{x_{0}}$ is orthonormal with respect to $g_{x_{0}}$, and let $d$ denote the distance on $M$ induced by $g$. In order to construct a metric with this property, one can start with an arbitrary metric $\tilde{g}$ on $M$. Then one takes a chart $(\phi, V)$ around $x_{0}$ and a scalar product $(\cdot, \cdot)$ on $\mathbb{R}^{d}$ such that $B_{x_{0}}$ is orthonormal with respect to the induced scalar product $\left(D \phi_{x_{0}} \cdot D \phi_{x_{0}} \cdot\right)$ on $T_{x_{0}} M$. On $V$ consider the pullback $\hat{g}$ of $(\cdot, \cdot)$ by $\phi$, i.e.,

$$
\hat{g}(x)(v, w):=\left(D \phi_{x} v, D \phi_{x} w\right) \text { for all } x \in V, v, w \in T_{x} M .
$$

Then let $\theta: M \rightarrow[0,1]$ be a smooth cut-off function such that $\operatorname{supp} \theta \subset V$ and $\theta(x)=1$ on a compact neighborhood $W$ of $x_{0}$. Define $g$ by

$$
g(x):=\left\{\begin{array}{cl}
\theta(x) \hat{g}(x)+(1-\theta(x)) \tilde{g}(x) & \text { for all } x \in V, \\
\tilde{g}(x) & \text { for all } x \in M \backslash V .
\end{array}\right.
$$

It can easily be seen that $g$ is a Riemannian metric on $M$ with $g_{x_{0}}$ having the desired property.

Step 2: We fix some constants: Let $S_{0}$ be a real number which satisfies

$$
S_{0}>\sum_{j=1}^{r} d_{j} \max \left\{0, \rho_{j}\right\} .
$$

Choose $\xi=\xi\left(S_{0}\right)>0$ such that

$$
0<d \xi<S_{0}-\sum_{j=1}^{r} d_{j} \max \left\{0, \rho_{j}\right\} .
$$

Let $\delta \in(0, \rho)$ be chosen small enough such that $\rho_{j}<0$ implies $\rho_{j}+\delta<0$ for all $j \in\{1, \ldots, r\}$. From Lemma 4.3 it follows that there exists a constant $c=c(\delta)>0$ such that

$$
\forall j \in\{1, \ldots, r\}: \forall k \in \mathbb{N}_{0}:\left\|e^{k T_{0} R_{j}}\right\| \leq c e^{\left(\rho_{j}+\delta\right) k T_{0}},
$$

where $\|\cdot\|$ denotes the operator norm on $\operatorname{Hom}\left(T_{x_{0}} M, T_{x_{0}} M\right)$ induced by $g_{x_{0}}$. For every $t>0$ we define positive integers

$$
M_{j}(t):=\left\{\begin{array}{cc}
\left\lfloor e^{\left(\rho_{j}+\xi\right) t}\right\rfloor+1 & \text { if } \rho_{j} \geq 0 \\
1 & \text { if } \rho_{j}<0
\end{array}, j=1, \ldots, r\right.
$$

Moreover, we define a function $\beta:(0, \infty) \rightarrow(0, \infty)$ by

$$
\beta(t):=c \sqrt{r} \max _{1 \leq j \leq r}\left[e^{\left(\rho_{j}+\delta\right) t} \frac{\sqrt{d_{j}}}{M_{j}(t)}\right] .
$$

If $\rho_{j}<0$, then (by our definitions) $\rho_{j}+\delta<0$ and $M_{j}(t) \equiv 1$. This implies that $e^{\left(\rho_{j}+\delta\right) t} \frac{\sqrt{d_{j}}}{M_{j}(t)}$ converges to zero for $t \rightarrow \infty$. If $\rho_{j} \geq 0$, we have $M_{j}(t) \geq e^{\left(\rho_{j}+\xi\right) t}$ by (4.8) and hence

$$
e^{\left(\rho_{j}+\delta\right) t} \frac{\sqrt{d_{j}}}{M_{j}(t)} \leq e^{\left(\rho_{j}+\delta\right) t} \frac{\sqrt{d_{j}}}{e^{\left(\rho_{j}+\xi\right) t}}=\sqrt{d_{j}} e^{(\delta-\xi) t}
$$


Since $\delta \in(0, \xi)$, we have $\delta-\xi<0$ and hence the term above converges to zero for $t \rightarrow \infty$. Thus, also $\beta(t) \rightarrow 0$ for $t \rightarrow \infty$. This implies that for given $\varepsilon>0$ we can choose a number $\tau=2 k T_{0}$ with $k \in \mathbb{N}$ big enough such that

$$
\beta(\tau)<1 \text { and } \frac{d}{\tau} \ln (2)<\varepsilon .
$$

Since we assume that the linearization along $\left(\varphi\left(\cdot, x_{0}, u_{0}\right), u_{0}\right)$ is controllable, by Proposition 3.5 there exists a constant $C>0$ with the following property (Note that controllability on $\left[0, T_{0}\right]$ implies controllability on $\left.[0, \tau]\right)$ :

$$
\forall \lambda \in T_{x_{0}} M: \exists \mu \in L^{\infty}\left([0, \tau], \mathbb{R}^{m}\right): \varphi^{l}(\tau, \lambda, \mu)=0 \text { and }\|\mu\|_{[0, \tau]} \leq C\|\lambda\| .
$$

Let $W_{1} \subset T_{x_{0}} M$ and $W_{2} \subset M$ be open neighborhoods of $0 \in T_{x_{0}} M$ and $x_{0}$, respectively, such that

$$
\exp _{x_{0}}: W_{1} \rightarrow W_{2}
$$

is a diffeomorphism. The inverse of $\left.\exp _{x_{0}}\right|_{W_{1}}$ will simply be denoted by $\exp _{x_{0}}^{-1}$. Now, choose $b_{0}>0$ small enough that the following conditions are satisfied:

$$
\left\{\begin{array}{l}
\operatorname{cl} B_{b_{0}}(0) \subset W_{1}, \\
\operatorname{cl} B_{b_{0}}\left(x_{0}\right) \subset D, \\
\operatorname{cl} B_{C \sqrt{d} b_{0}}\left(u_{0}(t)\right) \subset U \text { for almost all } t \in\left[0, T_{0}\right], \\
\varphi\left(\tau, \operatorname{cl} B_{b_{0}}\left(x_{0}\right), u\right) \subset W_{2} \text { if }\left\|u-u_{0}\right\|_{[0, \tau]} \leq C \sqrt{d} b_{0} .
\end{array}\right\}
$$

The second and third inclusion are possible, since $x_{0} \in \operatorname{int} D$ and $u_{0}(t)$ is contained in a compact subset of int $U$ for almost all $t \in\left[0, T_{0}\right]$. The last one is possible by continuity of $(x, u) \mapsto \varphi(\tau, x, u)$ (see Proposition 3.2(i)). By Proposition 3.3 there exists a function $\zeta=\zeta_{\tau, \sqrt{d} C}:[0, \alpha) \rightarrow \mathbb{R}_{0}^{+}(\alpha>0)$ with

$$
\left\|\exp _{x_{0}}^{-1}(\varphi(\tau, x, u))-\varphi^{l}\left(\tau, \exp _{x_{0}}^{-1}(x), u-u_{0}\right)\right\| \leq \zeta(b) b
$$

for all $(x, u) \in M \times \mathcal{U}$ with $d\left(x, x_{0}\right) \leq b \leq b_{0}$ and $\left\|u-u_{0}\right\|_{[0, \tau]} \leq C \sqrt{d} b$, and $\zeta(b) \rightarrow 0$ for $b \rightarrow 0$. We can assume that $b_{0}<\alpha$ and hence $\zeta\left(b_{0}\right)$ is defined. Because of the strict inequality $\beta(\tau)<1$ we can also assume that $b_{0}$ is chosen small enough that

$$
\sqrt{r} \zeta\left(b_{0}\right)+\beta(\tau) \leq 1 .
$$

Step 3: By Lemma 4.2 and 4.12 we can assume that $K=\operatorname{cl} B_{b_{0}}\left(x_{0}\right)$. Consider a $d$-dimensional cube $\mathcal{C}$ in $T_{x_{0}} M$ centered at the origin with sides of length $2 b_{0}$ parallel to the vectors of the orthonormal basis $B_{x_{0}}$. Then $\exp _{x_{0}}^{-1}(K)=\operatorname{cl} B_{b_{0}}(0) \subset T_{x_{0}} M$ and hence $\exp _{x_{0}}^{-1}(K) \subset \mathcal{C}$. Partition $\mathcal{C}$ by dividing each coordinate axis corresponding to a component of the $j^{\text {th }}$ Lyapunov space of $R$ into $M_{j}(\tau)$ intervals of equal length. The total number of subcuboids in this partition is $\prod_{j=1}^{r} M_{j}(\tau)^{d_{j}}$. Now pick an arbitrary $x \in \operatorname{cl} B_{b_{0}}\left(x_{0}\right)$. Let $\gamma_{0}:[0,1] \rightarrow M$ be a shortest geodesic from $x_{0}$ to $x$ and let $\lambda_{x} \in \mathcal{C}$ be the center of a subcuboid which contains $\exp _{x_{0}}^{-1}(x)=\dot{\gamma}_{0}(0)$. (Note that $\left\|\dot{\gamma}_{0}(0)\right\|=\mathcal{L}\left(\gamma_{0}\right)=d\left(x_{0}, x\right) \leq b_{0}$.) Then the following estimate holds, where the additional superscripts denote components of vectors within the corresponding Lyapunov spaces of $R$ :

$$
\begin{aligned}
\left\|\dot{\gamma}_{0}(0)^{(j)}-\lambda_{x}^{(j)}\right\| & =\left[\sum_{l=1}^{d_{j}}\left(\dot{\gamma}_{0}(0)^{(j, l)}-\lambda_{x}^{(j, l)}\right)^{2}\right]^{1 / 2} \\
& \leq\left[\sum_{l=1}^{d_{j}}\left(\frac{b_{0}}{M_{j}(\tau)}\right)^{2}\right]^{1 / 2}=\frac{\sqrt{d_{j}}}{M_{j}(\tau)} b_{0} .
\end{aligned}
$$


By 4.11 there exists $u_{x} \in L^{\infty}\left([0, \tau], \mathbb{R}^{m}\right)$ such that $\varphi^{l}\left(\tau, \lambda_{x}, u_{x}-u_{0}\right)=0$ or equivalently,

$$
\varphi^{l}\left(\tau, \lambda_{x}, u_{x}\right)=\varphi^{l}\left(\tau, 0, u_{0}\right)
$$

and

$$
\left\|u_{x}-u_{0}\right\|_{[0, \tau]} \leq C\left\|\lambda_{x}\right\| \leq C\left[\sum_{j=1}^{r} \sum_{l=1}^{d_{j}}\left\|\lambda_{x}^{(j, l)}\right\|^{2}\right]^{1 / 2} \leq C \sqrt{d} b_{0},
$$

since $\lambda_{x} \in \mathcal{C}$ implies $\left\|\lambda_{x}^{(j, l)}\right\| \leq b_{0}$ for each component. By 4.12 it holds that $u_{x} \in \mathcal{U}$ and

$$
\varphi\left(\tau, x, u_{x}\right) \in W_{2} .
$$

Let $\gamma_{1}:[0,1] \rightarrow M$ be a shortest geodesic from $x_{0}$ to $\varphi\left(\tau, x, u_{x}\right)$. Then

$$
d\left(\varphi\left(\tau, x, u_{x}\right), x_{0}\right)=\mathcal{L}\left(\gamma_{1}\right)=\int_{0}^{1} \underbrace{\left\|\dot{\gamma}_{1}(t)\right\|}_{=\text {constant }} d t=\left\|\dot{\gamma}_{1}(0)\right\| .
$$

By the triangle inequality we have

$$
\left\|\dot{\gamma}_{1}(0)^{(j)}\right\| \leq\left\|\dot{\gamma}_{1}(0)^{(j)}-\varphi^{l}\left(\tau, \dot{\gamma}_{0}(0), u_{x}-u_{0}\right)^{(j)}\right\|+\left\|\varphi^{l}\left(\tau, \dot{\gamma}_{0}(0), u_{x}-u_{0}\right)^{(j)}\right\| .
$$

Since $g$ is chosen such that the Lyapunov spaces of $R$ are orthogonal, for the first term we obtain

$$
\begin{aligned}
\| \dot{\gamma}_{1}(0)^{(j)} & -\varphi^{l}\left(\tau, \dot{\gamma}_{0}(0), u_{x}-u_{0}\right)^{(j)}\|=\|\left[\dot{\gamma}_{1}(0)-\varphi^{l}\left(\tau, \dot{\gamma}_{0}(0), u_{x}-u_{0}\right)\right]^{(j)} \| \\
& \leq\left\|\dot{\gamma}_{1}(0)-\varphi^{l}\left(\tau, \dot{\gamma}_{0}(0), u_{x}-u_{0}\right)\right\| \\
& =\left\|\exp _{x_{0}}^{-1}\left(\varphi\left(\tau, x, u_{x}\right)\right)-\varphi^{l}\left(\tau, \exp _{x_{0}}^{-1}(x), u_{x}-u_{0}\right)\right\| \stackrel{\text { 4.13 }}{\leq} \zeta\left(b_{0}\right) b_{0} .
\end{aligned}
$$

By linearity of $\varphi^{l}(\tau, \cdot, \cdot)$ for the second term we get

$$
\begin{aligned}
\left\|\varphi^{l}\left(\tau, \dot{\gamma}_{0}(0), u_{x}-u_{0}\right)^{(j)}\right\| & =\left\|\varphi^{l}\left(\tau, \dot{\gamma}_{0}(0), u_{x}\right)^{(j)}-\varphi^{l}\left(\tau, 0, u_{0}\right)^{(j)}\right\| \\
& =\left\|\varphi^{l}\left(\tau, \dot{\gamma}_{0}(0)-\lambda_{x}, 0\right)^{(j)}\right\| \\
& =\varphi^{l}\left(\tau, \dot{\gamma}_{0}(0), u_{x}\right)^{(j)}-\varphi^{l}\left(\tau, \lambda_{x}, u_{x}\right)^{(j)} \| \\
& \stackrel{4.4}{=}\left\|\left[e^{2 k T_{0} R}\left(\dot{\gamma}_{0}(0)-\lambda_{x}\right)\right]^{(j)}\right\| \\
& =\left\|\left[e^{\tau R}\left(\dot{\gamma}_{0}(0)-\lambda_{x}\right)\right]^{(j)}\right\| .
\end{aligned}
$$

By invariance of the Lyapunov spaces of $R$ under $e^{\tau R}$ we obtain

$$
\begin{aligned}
\left\|\varphi^{l}\left(\tau, \dot{\gamma}_{0}(0), u_{x}-u_{0}\right)^{(j)}\right\| & =\left\|e^{\tau R}\left(\dot{\gamma}_{0}(0)-\lambda_{x}\right)^{(j)}\right\| \\
& \leq\left\|e^{\tau R_{j}}\right\|\left\|\left(\dot{\gamma}_{0}(0)-\lambda_{x}\right)^{(j)}\right\| \\
& \stackrel{4.7)}{\leq} c e^{\left(\rho_{j}+\delta\right) \tau}\left\|\left(\dot{\gamma}_{0}(0)-\lambda_{x}\right)^{(j)}\right\| .
\end{aligned}
$$


Altogether, we have

$$
\begin{gathered}
\left\|\dot{\gamma}_{1}(0)^{(j)}\right\| \leq \zeta\left(b_{0}\right) b_{0}+c e^{\left(\rho_{j}+\delta\right) \tau}\left\|\left(\dot{\gamma}_{0}(0)-\lambda_{x}\right)^{(j)}\right\| \\
\stackrel{4.15)}{\leq} \zeta\left(b_{0}\right) b_{0}+c e^{\left(\rho_{j}+\delta\right) \tau} \frac{\sqrt{d_{j}}}{M_{j}(\tau)} b_{0} .
\end{gathered}
$$

By orthogonality of the Lyapunov spaces of $R$ it follows that

$$
\begin{aligned}
d\left(\varphi\left(\tau, x, u_{x}\right), x_{0}\right) & =\left\|\dot{\gamma}_{1}(0)\right\|=\left(\sum_{j=1}^{r}\left\|\dot{\gamma}_{1}(0)^{(j)}\right\|^{2}\right)^{1 / 2} \\
& \leq\left(\sum_{j=1}^{r}\left(\zeta\left(b_{0}\right) b_{0}+c e^{\left(\rho_{j}+\delta\right) \tau} \frac{\sqrt{d_{j}}}{M_{j}(\tau)} b_{0}\right)^{2}\right)^{1 / 2} \\
& \stackrel{(\Delta)}{\leq} \sqrt{r} \zeta\left(b_{0}\right) b_{0}+\left(\sum_{j=1}^{r}\left(c e^{\left(\rho_{j}+\delta\right) \tau} \frac{\sqrt{d_{j}}}{M_{j}(\tau)} b_{0}\right)^{2}\right)^{1 / 2} \\
& \leq \sqrt{r} \zeta\left(b_{0}\right) b_{0}+c \sqrt{r} \max _{1 \leq j \leq r}\left[e^{\left(\rho_{j}+\delta\right) \tau} \frac{\sqrt{d_{j}}}{M_{j}(\tau)}\right] b_{0} \\
& \stackrel{4.9}{=}\left[\sqrt{r} \zeta\left(b_{0}\right)+\beta(\tau)\right] b_{0} \stackrel{4.14}{\leq} b_{0} .
\end{aligned}
$$

The estimate $(\Delta)$ follows from the triangle inequality in $\mathbb{R}^{r}$. Hence, we have proved that $\prod_{j=1}^{r} M_{j}(\tau)^{d_{j}}$ admissible control functions are sufficient to steer from every initial value in $K$ back to $K$ in time $\tau$. By the no-return property of control sets it follows that the trajectories do not leave $Q$ within the time interval $(0, \tau)$. By iterated concatenation of these control functions we can construct an $(n \tau, K, Q)$-spanning set for each $n \in \mathbb{N}$ with $\left(\prod_{j=1}^{r} M_{j}(\tau)^{d_{j}}\right)^{n}$ elements and hence we obtain

$$
r_{\mathrm{inv}}^{*}(n \tau, K, Q) \leq\left(\prod_{j=1}^{r} M_{j}(\tau)^{d_{j}}\right)^{n} \stackrel{[4.8}{=}\left(\prod_{j: \rho_{j} \geq 0}\left(\left\lfloor e^{\left(\rho_{j}+\xi\right) \tau}\right\rfloor+1\right)^{d_{j}}\right)^{n},
$$

which implies

$$
\begin{aligned}
h_{\mathrm{inv}}^{*}(K, Q) & =\limsup _{n \rightarrow \infty} \frac{1}{n \tau} \ln r_{\mathrm{inv}}^{*}(n \tau, K, Q) \leq \frac{1}{\tau} \sum_{j: \rho_{j} \geq 0} \ln \left(\left\lfloor e^{\left(\rho_{j}+\xi\right) \tau}\right\rfloor+1\right)^{d_{j}} \\
& =\sum_{j: \rho_{j} \geq 0} d_{j} \frac{1}{\tau} \ln \left(\left\lfloor e^{\left(\rho_{j}+\xi\right) \tau}\right\rfloor+1\right) \leq \sum_{j: \rho_{j} \geq 0} d_{j} \frac{1}{\tau} \ln \left(2 e^{\left(\rho_{j}+\xi\right) \tau}\right) \\
& =\sum_{j: \rho_{j} \geq 0} d_{j}\left(\frac{\ln (2)}{\tau}+\left(\rho_{j}+\xi\right)\right) \leq \frac{d}{\tau} \ln (2)+d \xi+\sum_{j: \rho_{j}>0} d_{j} \rho_{j} \\
& \stackrel{4.6}{<} \frac{d}{\tau} \ln (2)+S_{0} \stackrel{4.10}{<} S_{0}+\varepsilon .
\end{aligned}
$$

The first equality follows from Proposition 2.1. Since $\varepsilon$ can be chosen arbitrarily small and $S_{0}$ arbitrarily close to $\sum_{j=1}^{r} d_{j} \max \left\{0, \rho_{j}\right\}$, the assertion of the theorem follows. (

REMARK 4.1. We do not know if periodicity of the controlled trajectory in our 
main theorem is an essential assumption or if it can be weakened. But we think that some regularity property will be necessary.

REMARK 4.2. If $D$ is a control set of the control-affine system 2.1) with nonvoid interior and if the system is locally accessible on int D, then from [5, Prop. 4.3.3] it follows that there exist controlled periodic trajectories in int D. But in general it is not clear if the linearization along any of those trajectories is controllable.

Since an equilibrium is a periodic solution for every period $T_{0}>0$, and the corresponding Lyapunov exponents are the real parts of the eigenvalues of the linearization at the equilibrium, the following corollary immediately follows.

COROllary 4.5. Consider the control-affine system (2.1) and let $g$ be a Riemannian metric on $M$. Let $D \subset M$ be a control set with nonvoid interior and compact closure $Q=\operatorname{cl} D$. Let $\left(x_{0}, u_{0}\right) \in \operatorname{int} D \times \operatorname{int} U$ be an equilibrium pair with controllable linearization. Then for every compact set $K \subset D$ it holds that

$$
h_{\text {inv }}^{*}(K, Q) \leq \sum_{\lambda \in \sigma\left(\nabla F_{u_{0}}\left(x_{0}\right)\right)} \max \{0, \operatorname{Re}(\lambda)\}
$$

where every eigenvalue $\lambda$ is counted with its multiplicity.

For one-dimensional control-affine systems with a single control vector field we can derive a formula for the invariance entropy of a control set from Corollary 4.5 and Theorem 14 in [7]. Moreover, here we can show that $h_{\mathrm{inv}}(K, Q)$ and $h_{\mathrm{inv}}^{*}(K, Q)$ coincide, which is not clear at all in the general case, but can also be shown for control sets of linear systems (see [6, Theo. 4.2.4]).

COROLlary 4.6. Consider a control-affine system of the form

$$
\dot{x}(t)=f_{0}(x(t))+u(t) f_{1}(x(t)), \quad u \in \mathcal{U}
$$

on $M=\mathbb{R}$. Let $D$ be a bounded control set with nonvoid interior, and assume that local accessibility holds on $Q:=\mathrm{cl} D$. Then for every compact set $K \subset D$ with nonvoid interior we have

$$
h_{\mathrm{inv}}^{*}(K, Q)=h_{\mathrm{inv}}(K, Q)=\max \left\{0, \min _{x \in Q}\left[f_{0}^{\prime}(x)-\frac{f_{0}(x)}{f_{1}(x)} f_{1}^{\prime}(x)\right]\right\}
$$

Proof. The proof proceeds in three steps.

Step 1: By [5, Lem. 3.2.13] $D$ is connected and thus $Q$ is a compact interval. In order to show that formula 4.21 makes sense, we have to prove that $f_{1}(x) \neq 0$ for all $x \in Q$ : Assume to the contrary that $f_{1}\left(x^{*}\right)=0$ for some $x^{*} \in Q$. From [5. Theo. 8.1.1] it follows that for every $x \in Q$ there exists $u_{x} \in U$ with $f_{0}(x)+u_{x} f_{1}(x)=0$. Hence, $f_{0}\left(x^{*}\right)=0$, which implies $\varphi\left(t, x^{*}, u\right)=x^{*}$ for all $t \in \mathbb{R}$ and $u \in \mathcal{U}$ and therefore contradicts local accessibility on $Q$.

Step 2: Now we prove the lower bound, using [7, Theo. 14]. To this end, we define a volume form on a small open neighborhood $W$ of $Q$ by

$$
\omega(x):=f_{1}(x)^{-1} \cdot \omega_{0}(x)
$$

where $\omega_{0}$ denotes the standard volume form. Since $f_{1}(x) \neq 0$ on $Q$ implies $f_{1}(x) \neq 0$ on a neighborhood of $Q$, the definition of $\omega$ makes sense. The divergence of the vector 
field $x \mapsto f_{0}(x)+u f_{1}(x)$ with respect to $\omega$ is (cp. [1, Prop. 6.5.17])

$$
\begin{aligned}
\operatorname{div}_{\omega}\left(f_{0}(x)+u f_{1}(x)\right) & =f_{0}^{\prime}(x)+u f_{1}^{\prime}(x)+\frac{\alpha^{\prime}(x)}{\alpha(x)}\left(f_{0}(x)+u f_{1}(x)\right) \\
& =f_{0}^{\prime}(x)+u f_{1}^{\prime}(x)-\frac{f_{1}^{\prime}(x)}{f_{1}(x)}\left(f_{0}(x)+u f_{1}(x)\right) \\
& =f_{0}^{\prime}(x)+u f_{1}^{\prime}(x)-\frac{f_{1}^{\prime}(x)}{f_{1}(x)} f_{0}(x)-u f_{1}^{\prime}(x) \\
& =f_{0}^{\prime}(x)-\frac{f_{0}(x)}{f_{1}(x)} f_{1}^{\prime}(x) .
\end{aligned}
$$

Hence, [7, Theo. 14] immediately implies

$$
h_{\text {inv }}(K, Q) \geq \max \left\{0, \min _{x \in Q}\left[f_{0}^{\prime}(x)-\frac{f_{0}(x)}{f_{1}(x)} f_{1}^{\prime}(x)\right]\right\} .
$$

Step 3: We prove the upper bound, using Corollary 4.5. To this end, let $x \in \operatorname{int} D$. Then, by [5, Theo. 8.1.1], there exists $u_{x} \in U$ such that $f_{0}(x)+u_{x} f_{1}(x)=0$. Since $f_{1}(x) \neq 0, u_{x}$ is unique, namely $u_{x}=-\frac{f_{0}(x)}{f_{1}(x)}$. By approximate controllability on $D$ there also exist $u_{ \pm} \in U$ such that $f_{0}(x)+u_{-} f_{1}(x)<0$ and $f_{0}(x)+u_{+} f_{1}(x)>0$, which implies that $u_{x}$ lies in the interior of the interval with endpoints $u_{-}$and $u_{+}$, and hence $u_{x} \in \operatorname{int} U$. The linearization of system (4.20) at the equilibrium pair $\left(x, u_{x}\right)$ is controllable, which in this case is equivalent to $f_{1}(x) \neq 0$ by the Kalman rank condition. Corollary 4.5 yields

$$
h_{\mathrm{inv}}^{*}(K, Q) \leq \max \left\{0, f_{0}^{\prime}(x)+u_{x} f_{1}^{\prime}(x)\right\}=\max \left\{0, f_{0}^{\prime}(x)-\frac{f_{0}(x)}{f_{1}(x)} f_{1}^{\prime}(x)\right\} .
$$

The point $x$ was chosen arbitrarily in int $D$ and thus we get

$$
\begin{aligned}
h_{\text {inv }}^{*}(K, Q) & \leq \inf _{x \in \operatorname{int} D} \max \left\{0, f_{0}^{\prime}(x)-\frac{f_{0}(x)}{f_{1}(x)} f_{1}^{\prime}(x)\right\} \\
& =\min _{x \in Q} \max \left\{0, f_{0}^{\prime}(x)-\frac{f_{0}(x)}{f_{1}(x)} f_{1}^{\prime}(x)\right\} \\
& =\max \left\{0, \min _{x \in Q}\left[f_{0}^{\prime}(x)-\frac{f_{0}(x)}{f_{1}(x)} f_{1}^{\prime}(x)\right]\right\} .
\end{aligned}
$$

Now, by 2.2 the assertion of the corollary follows.

REMARK 4.3. In the PhD thesis [6], an alternative proof for Corollary 4.6 can be found which does not use Theorem 4.4 in order to obtain the upper bound, but a combination of Lemma 4.2 and the upper bound proved in [7, Theorem 12].

5. Applications. In this section, we apply our results to projected bilinear control systems on the unit sphere.

EXAMPLE 5.1. Consider a bilinear control system

$$
\dot{x}(t)=\left[A_{0}+\sum_{i=1}^{m} u_{i}(t) A_{i}\right] x(t), \quad u \in \mathcal{U},
$$

on $\mathbb{R}^{d}$ and its projection to the unit sphere $S^{d-1}=\left\{x \in \mathbb{R}^{d}:\|x\|=1\right\}$,

$$
\dot{s}(t)=F_{u(t)}(s(t)), \quad F_{u}(s)=F(s, u)=\left(A(u)-s^{T} A(u) s I\right) s, \quad u \in \mathcal{U} .
$$


Let $D \subset S^{d-1}$ be a control set of the projected system with nonvoid interior and set $Q:=\operatorname{cl} D$. Since $S^{d-1}$ is compact, also $Q$ is compact. In [7], we derived the following formula for the covariant derivative of $F_{u}$ :

$$
\nabla F_{u}(s)=Q_{s}\left(A(u)-s^{T} A(u) s I\right), \quad Q_{s}=I-s s^{T} .
$$

A pair $\left(s_{0}, u_{0}\right) \in \operatorname{int} D \times \operatorname{int} U$ is an equilibrium pair if and only if

$$
0=F\left(s_{0}, u_{0}\right)=A\left(u_{0}\right) s_{0}-\left(s_{0}^{T} A\left(u_{0}\right) s_{0}\right) s_{0},
$$

i.e., if and only if $s_{0}$ is an eigenvector of $A\left(u_{0}\right)$. We write $\lambda$ for the corresponding eigenvalue $s_{0}^{T} A\left(u_{0}\right) s_{0}$. We want to compute the spectrum of $\nabla F_{u_{0}}\left(s_{0}\right)$. To this end, let $v \in T_{s_{0}} S^{d-1}=s_{0}^{\perp}$. Then

$$
\nabla F_{u_{0}}\left(s_{0}\right) v=Q_{s_{0}}\left(A\left(u_{0}\right) v-\left(s_{0}^{T} A\left(u_{0}\right) s_{0}\right) v\right)=Q_{s_{0}} A\left(u_{0}\right) v-\lambda v .
$$

Hence, it suffices to determine the eigenvalues of the linear map $L:=\left.Q_{s_{0}} A\left(u_{0}\right)\right|_{T_{s_{0}} S^{d-1}}$. To this end, let $\mu \in \mathbb{C}$ be an arbitrary eigenvalue of $A\left(u_{0}\right)$ and $z \notin\left\langle s_{0}\right\rangle$ a corresponding (complex) eigenvector. Consider the vector $\zeta:=Q_{s_{0}} z \in T_{s_{0}} S^{d-1} \oplus i T_{s_{0}} S^{d-1}$. Then we obtain

$$
\begin{aligned}
L \zeta & =Q_{s_{0}} A\left(u_{0}\right) Q_{s_{0}} z=Q_{s_{0}} A\left(u_{0}\right)\left(I-s_{0} s_{0}^{T}\right) z=Q_{s_{0}} A\left(u_{0}\right) z-Q_{s_{0}} A\left(u_{0}\right) s_{0} s_{0}^{T} z \\
& =\mu Q_{s_{0}} z-Q_{s_{0}}\left(A\left(u_{0}\right) s_{0}\right)\left(s_{0}^{T} z\right)=\mu \zeta-Q_{s_{0}}\left(\lambda s_{0}\right)\left(s_{0}^{T} z\right) \\
& =\mu \zeta-\lambda\left(s_{0}^{T} z\right) Q_{s_{0}} s_{0}=\mu \zeta .
\end{aligned}
$$

Hence, the eigenvalues of $L$ coincide with eigenvalues of $A\left(u_{0}\right)$ and the eigenvalues of $\nabla F_{u_{0}}\left(s_{0}\right)$ are the eigenvalues of $A\left(u_{0}\right)$ minus $\lambda$. Therefore, under the assumption that the linearization at $\left(s_{0}, u_{0}\right)$ is controllable, by Corollary 4.5 the estimate

$$
h_{\mathrm{inv}}^{*}(K, Q) \leq \sum_{j=1}^{d-1} \max \left\{0,\left(\operatorname{Re}\left(\mu_{j}\right)-\lambda\right)\right\}
$$

holds true for every compact set $K \subset D$, where $\lambda, \mu_{1}, \ldots, \mu_{d-1}$ are the eigenvalues of $A\left(u_{0}\right)$.

REMARK 5.1. By [5, Theo. 7.3.3], for the projected system on projective space $\mathbb{P}^{d-1}$ there exist (finitely many) control sets with nonvoid interior under the assumption of local accessibility. The connected components of the lifts of these control sets to the unit sphere are control sets for the system on the sphere.

EXAMPLE 5.2. Consider a bilinear control system on $\mathbb{R}^{2}$ of the form

$$
\dot{x}(t)=\left(A_{0}+u(t) A_{1}\right) x(t), \quad u \in \mathcal{U} .
$$

Let $A_{0}=\left(a_{i j}^{0}\right), A_{1}=\left(a_{i j}^{1}\right)$, and $A(u)=A_{0}+u A_{1}$. Consider the projection of 5.1) to the unit circle $S^{1}$, given by

$$
\dot{s}(t)=\left(A(u(t))-s(t)^{T} A(u(t)) s(t) I\right) s(t), \quad u \in \mathcal{U} .
$$

Let $D \subset S^{1}$ be a control set of system (5.2) with nonvoid interior, which is not the whole circle, and assume that local accessibility holds on $Q:=\mathrm{cl} D$. We want to compute $h_{\mathrm{inv}}^{*}(K, Q)$ for every compact set $K \subset D$ with nonvoid interior. To this end, 
we describe system (5.2) in polar coordinates. By writing $s(t)=(\cos \varphi(t), \sin \varphi(t)) a$ simple calculation leads to the system

$$
\dot{\varphi}(t)=f_{0}(\varphi(t))+u(t) f_{1}(\varphi(t)), \quad u \in \mathcal{U},
$$

where $f_{0}, f_{1}:[0,2 \pi) \rightarrow \mathbb{R}$ are given by

$$
f_{k}(\varphi)=\left(a_{22}^{k}-a_{11}^{k}\right) \sin \varphi \cos \varphi-a_{12}^{k} \sin ^{2} \varphi+a_{21}^{k} \cos ^{2} \varphi, \quad k=0,1 .
$$

For the derivatives $f_{k}^{\prime}$ we get

$$
f_{k}^{\prime}(\varphi)=\left(a_{22}^{k}-a_{11}^{k}\right) \cos (2 \varphi)-\left(a_{12}^{k}+a_{21}^{k}\right) \sin (2 \varphi) .
$$

By Corollary 4.6 we obtain that $h_{\mathrm{inv}}^{*}(K, Q)$ is the maximum of 0 and the minimum of the following function on $Q$ :

$$
\begin{aligned}
\varphi & \mapsto\left(a_{22}^{0}-a_{11}^{0}\right) \cos (2 \varphi)-\left(a_{12}^{0}+a_{21}^{0}\right) \sin (2 \varphi) \\
& -\frac{\left[\left(a_{22}^{0}-a_{11}^{0}\right) \sin \varphi \cos \varphi-a_{12}^{0} \sin ^{2} \varphi+a_{21}^{0} \cos ^{2} \varphi\right]\left[\left(a_{22}^{1}-a_{11}^{1}\right) \cos (2 \varphi)-\left(a_{12}^{1}+a_{21}^{1}\right) \sin (2 \varphi)\right]}{\left(a_{22}^{1}-a_{11}^{1}\right) \sin \varphi \cos \varphi-a_{12}^{1} \sin ^{2} \varphi+a_{21}^{1} \cos ^{2} \varphi}
\end{aligned}
$$

The next example provides an application of this formula.

EXAMPLE 5.3. We consider the scalar second-order system

$$
\ddot{y}(t)+2 b \dot{y}(t)-(1+u(t)) y(t)=0, \quad u \in \mathcal{U},
$$

with $b>0$ and control range $U=[-\rho, \rho]$, where $0<\rho<b^{2}+1$. This equation describes the linearization of a controlled damped mathematical pendulum at the unstable position (a linear oscillator). The corresponding first-order system is the following bilinear control system:

$$
\dot{x}(t)=\underbrace{\left(\begin{array}{rr}
0 & 1 \\
1 & -2 b
\end{array}\right)}_{=: A_{0}} x(t)+u(t) \underbrace{\left(\begin{array}{ll}
0 & 0 \\
1 & 0
\end{array}\right)}_{=: A_{1}} x(t), \quad u \in \mathcal{U} .
$$

The eigenvalues of the matrix $A_{0}$ are given by

$$
\lambda_{ \pm}=-b \pm \sqrt{b^{2}+1}
$$

Since $b>0, \lambda_{-}$is negative and $\lambda_{+}$is positive. Hence, the uncontrolled system has one stable and one unstable direction. From the preceding example it follows that the projected system on $S^{1}$ is given by

$$
\dot{\varphi}=\left(-2 b \sin \varphi \cos \varphi-\sin ^{2} \varphi+\cos ^{2} \varphi\right)+u(t) \cos ^{2} \varphi, \quad u \in \mathcal{U} .
$$

From [5, Theo. 8.1.1] it follows that the control sets on $S^{1}$ consist of equilibria. Hence, in order to determine these sets, we have to find the zeros of the right-hand side. To this end, we divide by $\cos ^{2} \varphi$ (which is possible for $\varphi \notin\left\{\frac{\pi}{2}, \frac{3 \pi}{2}\right\}$ ). This yields

$$
\tan ^{2} \varphi+2 b \tan \varphi-(1+u)=0 \Leftrightarrow \tan \varphi=-b \pm \sqrt{b^{2}+1+u} .
$$

Hence, we obtain the solutions

$$
\varphi_{1, \pm}=\arctan \left(-b \pm \sqrt{b^{2}+1+u}\right) \in\left(-\frac{\pi}{2}, \frac{\pi}{2}\right)
$$


and, by $\pi$-periodicity of the tangent function,

$$
\varphi_{2, \pm}=\pi+\arctan \left(-b \pm \sqrt{b^{2}+1+u}\right) \in\left(\frac{\pi}{2}, \frac{3 \pi}{2}\right)
$$

The solutions are real numbers, since

$$
b^{2}+1+u \in\left[b^{2}+1-\rho, b^{2}+1+\rho\right] \subset\left(0,2\left(b^{2}+1\right)\right) .
$$

Hence, in $\left(-\frac{\pi}{2}, \frac{\pi}{2}\right)$ we obtain the following two intervals of equilibria, which are the closures of control sets:

$$
\begin{aligned}
& Q_{1,-}=\left[\arctan \left(-b-\sqrt{b^{2}+1+\rho}\right), \arctan \left(-b-\sqrt{b^{2}+1-\rho}\right)\right], \\
& Q_{1,+}=\left[\arctan \left(-b+\sqrt{b^{2}+1-\rho}\right), \arctan \left(-b+\sqrt{b^{2}+1+\rho}\right)\right],
\end{aligned}
$$

and in $\left(\frac{\pi}{2}, \frac{3 \pi}{2}\right)$ we obtain the sets $Q_{2, \pm}=\pi+Q_{1, \pm}$. Applying the result from the preceding example we can calculate the invariance entropy of these control sets. An elementary computation gives

$$
\left.h_{\text {inv }}^{(*)}\left(K, Q_{i, \pm}\right)=\max \left\{0, \min _{\varphi \in Q_{i, \pm}}(-2 b-2 \tan \varphi)\right)\right\}, \quad i=1,2 .
$$

Hence, we obtain

$$
\begin{aligned}
& h_{\text {inv }}^{(*)}\left(K, Q_{i,-}\right)=\max \left\{0, \min _{u \in[-\rho, \rho]}\left(2 \sqrt{b^{2}+1-u}\right)\right\}=2 \sqrt{b^{2}+1-\rho}, \\
& h_{\text {inv }}^{(*)}\left(K, Q_{i,+}\right)=0 .
\end{aligned}
$$

The sets $Q_{i,+}$ are easily seen to be invariant control sets, while the sets $Q_{i,-}$ are the closures of open, variant control sets. The following figure illustrates the situation.

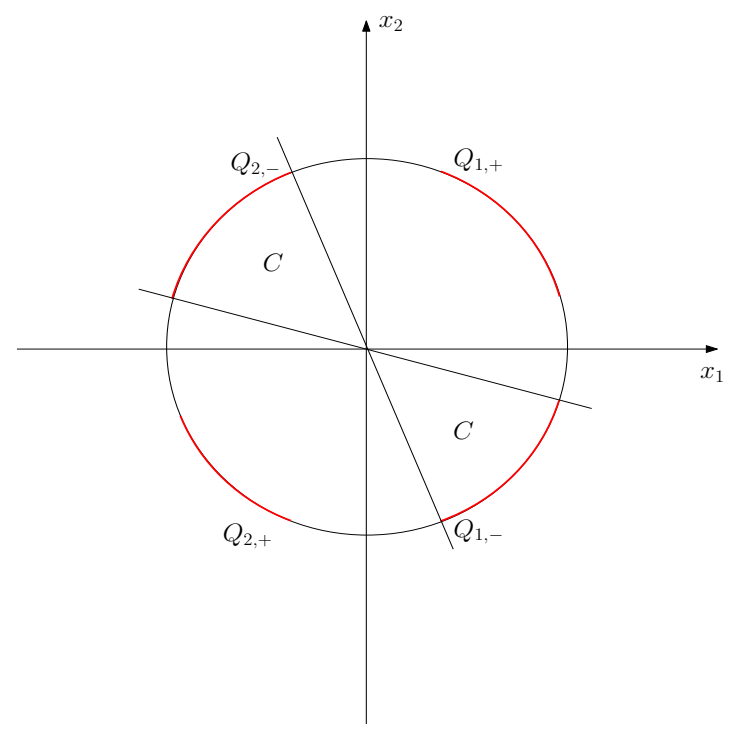

FIG. 5.1. The control sets on the unit circle 
The set $Q_{1,-}$ contains the point $\varphi_{0}=\arctan \left(-b-\sqrt{b^{2}+1}\right)$, which is an equilibrium for the constant control $u=0$, i.e., the vectors $\pm\left(\cos \left(\varphi_{0}\right), \sin \left(\varphi_{0}\right)\right)$ are eigenvectors of $A_{0}$ corresponding to the stable eigenvalue $\lambda_{-}=-b-\sqrt{b^{2}+1}$. On $\operatorname{int} Q_{1,-}$ and int $Q_{2,-}$ the projected system is controllable. This implies that from any point in the interior of the cone over $Q_{1,-}$ and $Q_{2,-}, C:=\pi^{-1}\left(Q_{1,-} \cup Q_{2,-}\right)$, where

$$
\pi(x)=\frac{x}{\|x\|}, \quad \pi: \mathbb{R}^{2} \backslash\{0\} \rightarrow S^{1},
$$

it is possible to steer to the stable axis, i.e., the eigenspace of $A_{0}$ corresponding to $\lambda_{-}$. Hence, here it is possible to stabilize the system. It is easily seen that outside of $C$ stabilization is not possible.

6. Acknowledgements. The paper is, essentially, a main part of the author's $\mathrm{PhD}$ thesis. I like to thank Prof. Fritz Colonius, my $\mathrm{PhD}$ advisor, for many fruitful discussions.

\section{REFERENCES}

[1] R. Abraham, J. E. Marsden, T. Ratiu, Manifolds, Tensor Analysis, and Applications, Second Edition, New York: Springer (1988).

[2] G. Bachman, L. Narici, Functional Analysis, New York: Academic Press (1966).

[3] C. Chicone, Ordinary Differential Equations with Applications, New York: Springer (1999).

[4] F. Colonius and C. Kawan, Invariance entropy for control systems, SIAM J. Control Optim. 48, 3 (2009), 1701-1721.

[5] F. Colonius and W. Kliemann, The Dynamics of Control, Birkhäuser-Verlag, Boston, 2000.

[6] C. Kawan, Invariance Entropy for Control Systems, Doctoral thesis, Institut für Mathematik, Universität Augsburg (2009).

[7] C. Kawan, Upper and Lower Estimates for Invariance Entropy, submitted (2009).

[8] C. Robinson, Dynamical Systems, Stability, Symbolic Dynamics, and Chaos, CRC Press: Boca Raton (1995).

[9] E. D. Sontag, Mathematical Control Theory, New York: Springer (1990).

[10] G. N. Nair, R. J. Evans, I. M. Y. Mareels, and W. Moran, Topological feedback entropy and nonlinear stabilization, IEEE Trans. Automat. Control 49, 9, (2004), 1585-1597. 